Pacific Journal of Mathematics

ARITHMETICAL PROPERTIES OF GENERALIZED 


\section{ARITHMETICAL PROPERTIES OF GENERALIZED RAMANUJAN SUMS}

Tom. M. Apostol

The sums studied in this paper are defined as follows. For any two arithmetical functions $f$ and $g$, let

$$
S_{f}, g(m, k)=\sum_{d \backslash(m, k)} f(d) g(k / d),
$$

where the sum extends over the divisors of the greatest common divisor $(m, k)$ of the positive integers $m$ and $k$. It should be noted that $m$ and $k$ do not enter symmetrically in (1) unless $g$ is constant.

The sums $S_{f, g}(m, k)$ generalize the Dirichlet convolution

$$
(f * g)(k)=\sum_{d \backslash k} f(d) g(k / d),
$$

to which they reduce when $(m, k)=k$. Multiplicative properties and finite Fourier expansions were obtained in [1]. A famous special case is Ramanujan's sum $c_{k}(m)$, the sum of the $m$ th powers of the primitive $k$ th roots of unity, for which we have

$$
c_{k}(m)=\sum_{\substack{h \text { mod } k \\(h, k)=1}} \exp (2 \pi i m h / k)=\sum_{d \mid\langle m, k)} d \mu(k / d),
$$

where $\mu$ is the Möbius function. The second sum in (3) is an example of (1) with $f(n)=n$ and $g(n)=\mu(n)$ for all $n$. When $(m, k)=1$ we have $c_{k}(m)=\mu(k)$, and when $(m, k)=k$ we have $c_{k}(m)=\varphi(m)$, Euler's totient.

In a study on cyclotomic polynomials, Hölder [4] showed that Ramanujan's sum can also be expressed in closed form as follows:

$$
c_{k}(m)=\frac{\varphi(m)}{\varphi(m /(k, m))} \mu(m /(k, m)) .
$$

The number on the right is called the Von Sterneck function and is denoted by $\Phi(m, k)$. Thus, (4) states that

$$
c_{k}(m)=\Phi(m, k) \text {. }
$$

The function $\Phi(m, k)$ was encountered by Von Sterneck in 1902 [11] in a study of restricted partitions with summands reduced to their least residues module $m$. Its properties were also studied by Nicol and Vandiver [7].

We derive further properties of the sums $S_{f, g}(m, k)$. Some of them 
generalize known properties of Ramanujan's sum or Von Sterneck's function. Others, when specialized, give new properties of these numbers. The methods of this paper are simpler than those used by earlier writers. In particular, roots of unity and restricted partitions $\bmod m$ play no role.

2. Properties of Dirichlet convolution. The Dirichlet convolution (2) provides a natural setting for the results of this paper. It is well known that the operation $*$ is commutative and associative and has the identity element $I$, where

$$
I(n)=\left[\frac{1}{n}\right]=\left\{\begin{array}{lll}
1 & \text { if } & n=1 \\
0 & \text { if } & n>1
\end{array}\right.
$$

If $g(1) \neq 0$ we let $g^{-1}$ denote the Dirichlet inverse of $g$, defined by the equation $g * g^{-1}=I$. In particular, $\mu^{-1}(n)=1$ for all $n$.

The set of all functions $g$ with $g(1) \neq 0$ forms an abelian group under the operation $*$. The subset of multiplicative functions is a subgroup. [A function $g$ is called multiplicative if $g(1)=1$ and if $g(m n)=g(m) g(n)$ whenever $(m, n)=1$.]

If $a$ is any arithmetical function and if $g(1) \neq 0$, the equation

$$
S(n)=\sum_{d \backslash n} a(d) g(n / d)
$$

holds if, and only if,

$$
a(n)=\sum_{d \mid n} S(d) g^{-1}(n / d)
$$

The special case with $g=\mu$ is the usual Möbius inversion formula.

By introducing the function

$$
\alpha_{n, d}=\left\{\begin{array}{lll}
1 & \text { if } & d \mid n \\
0 & \text { if } & d \nmid n,
\end{array}\right.
$$

we can rewrite the sum in (1) in two alternate forms:

$$
S_{f, g}(m, k)=\sum_{d \mid m} \alpha_{k, d} f(d) g(k / d),
$$

and

$$
S_{f, g}(m, k)=\sum_{d \mid k} \alpha_{m, d} f(d) g(k / d) .
$$

For fixed $k$, Equation (6) expresses $S_{f, g}(m, k)$ as a Dirichlet convolution,

$$
S_{f, g}(m, k)=\left(h_{k} * \mu^{-1}\right)(m),
$$

where 


$$
h_{k}(d)=\alpha_{k, d} f(d) g(k / d) \text {. }
$$

For fixed $m$, Equation (7) expresses $S_{f, g}(m, k)$ as another Dirichlet convolution,

$$
S_{f, g}(m, k)=\left(w_{m} * g\right)(k)
$$

where

$$
w_{m}(d)=\alpha_{m, d} f(d)
$$

3. Dirichlet convolutions involving $S_{f, g}(m, k)$. This section derives arithmetical identities in the form of divisor sums whose terms contain the numbers $S_{f, g}(m, k)$.

Notation. The function $\alpha$ is defined by (5) and, unless otherwise stated, the functions $f$ and $g$ are arbitrary. For any arithmetical function $a$, we denote the Dirichlet convolution $a * \mu^{-1}$ by $a^{*}$. Thus,

$$
a^{*}(n)=\sum_{d \mid n} a(d) \text {. }
$$

For example, we have $\mu^{*}=\mu * \mu^{-1}=I$, and $\left(\mu^{-1}\right)^{*}=\sigma$, where $\sigma(n)$ is the sum of the divisors of $n$.

THEOREM 1. If $n \geqq 1, k \geqq 1$ we have

$$
\sum_{d \mid n} S_{f, g}(d, k)=\sum_{d \mid(n, k)} f(d) g(k / d) \sigma(n / d) \text {. }
$$

Proof. For fixed $k$, let $S(m)=S_{f, g}(m, k)$. Then by (8) we have $S(m)=\left(h_{k} * \mu^{-1}\right)(m)$, so

$$
\begin{aligned}
\sum_{d \mid n} S_{f, g}(d, k) & =\sum_{d \mid n} S(d)=\left(S * \mu^{-1}\right)(n) \\
& =\left(h_{k} * \mu^{-1} * \mu^{-1}\right)(n)=\left(h_{k} * \sigma\right)(n) \\
& =\sum_{d \mid n} \alpha_{k, d} f(d) g(k / d) \sigma(n / d)=\sum_{d|n, d| k} f(d) g(k / d) \sigma(n / d),
\end{aligned}
$$

which proves Theorem 1.

EXAMPLES. Theorem 1 has a number of interesting corollaries. If $(n, k)=n$ we obtain

$$
\sum_{d \backslash n} S_{f, g}(d, k)=\sum_{d \backslash n} f(d) g(k / d) \sigma(n / d) .
$$

If $k=n$ this gives

$$
\sum_{d \backslash n} S_{f, g}(d, n)=\sum_{d \mid n} f(d) g(n / d) \sigma(n / d) .
$$

When $g=\mu$ we can write this as 


$$
\sum_{d \mid n} S_{f, \mu}(d, n)=\sum_{d \mid n} \mu(d) \sigma(d) f(n / d) .
$$

If $f$ is completely multiplicative, that is, if $f(a b)=f(a) f(b)$ for all $a, b$, and if $f(n) \neq 0$, then $f(n / d)=f(n) / f(d)$ and (11) gives us

$$
\begin{aligned}
\sum_{d \mid n} S_{f, \mu}(d, n) & =f(n) \sum_{d \mid n} \mu(d) \sigma(d) / f(d) \\
& =f(n) \prod_{p \nmid n}\left(1-\frac{\sigma(p)}{f(p)}\right)=f(n) \prod_{p \mid n}\left(1-\frac{2}{f(p)}\right),
\end{aligned}
$$

where the product is taken over all prime divisors of $n$. The special case $f(n)=n$ gives a formula of Nicol and Vandiver ([7], Theorem VIII),

$$
\sum_{d \backslash n} \Phi(d, n)=n \prod_{p \mid n}\left(1-\frac{2}{p}\right) .
$$

We also have the following more general result.

THEOREM 2. Let $a^{*}(n)=\sum_{d \mid n} a(d)$, where $a(n)$ is any arithmetical function. Then for $n \geqq 1, k \geqq 1$ we have

$$
\sum_{d \mid n} S_{f, g}(d, k) a(n / d)=\sum_{d \mid(n, k)} f(d) g(k / d) a^{*}(n / d) .
$$

Proof. With the notation used in the proof of Theorem 1 we have

$$
\begin{aligned}
\sum_{d \mid n} S_{f, g}(d, k) a(n / d) & =(S * a)(n)=\left(h_{k} * \mu^{-1} * a\right)(n)=\left(h_{k} * a^{*}\right)(n) \\
= & \sum_{d \backslash k} \alpha_{k, d} f(d) g(k / d) a^{*}(n / d)=\sum_{d|n, d| k} f(d) g(k / d) a^{*}(n / d),
\end{aligned}
$$

which proves Theorem 2.

ExAmples. When $a=\mu^{-1}$ then $a^{*}=\sigma$ and Theorem 2 reduces to Theorem 1. When $a=\mu$ then $a^{*}=I$ and the second sum in (13) is

$$
\sum_{d \mid(n, k)} f(d) g(k / d) a^{*}(n / d)=\sum_{d \mid(n, k)} f(d) g(k / d)[d / n]=\alpha_{k, n} f(n) g(k / n) .
$$

Hence (13) becomes

$$
\sum_{d \mid n} S_{f, g}(d, k) \mu(n / d)=\left\{\begin{array}{lll}
f(n) g(k / n) & \text { if } & n \mid k, \\
0 & \text { if } & n \nmid k
\end{array} .\right.
$$

This can also be deduced by Möbius inversion of (6) for fixed $k$.

When $k=n$, Equation (13) can be written as

$$
\sum_{d \backslash n} S_{f, g}(d, n) a(n / d)=\sum_{d \mid n} g(d) a^{*}(d) f(n / d) .
$$

If we take $g=\mu$ and assume that $a$ is multiplicative and that $f$ is 
completely multiplicative, with $f(n) \neq 0$, we obtain the following product formula, a further generalization of (12):

$$
\sum_{d \mid n} S_{f, \mu}(d, n) a(n / d)=f(n) \prod_{p \mid n}\left(1-\frac{a^{*}(p)}{f(p)}\right) .
$$

THEOREM 3. If $n \geqq 1, m \geqq 1$ we have

$$
\sum_{d \mid n} S_{f, g}(m, d)=\sum_{d \mid\langle m, n)} f(d) g^{*}(n / d),
$$

where $g^{*}(n)=\sum_{d \mid n} g(d)$.

Proof. For fixed $m$, let $S(k)=S_{f, g}(m, k)$. Then by (9) we have $S(k)=\left(w_{m} * g\right)(k)$, so

$$
\begin{aligned}
\sum_{d \backslash n} S_{f, g}(m, d) & =\sum_{d \backslash n} S(d)=\left(S * \mu^{-1}\right)(n) \\
& =\left(w_{m} * g * \mu^{-1}\right)(n)=\left(w_{m} * g^{*}\right)(n) \\
& =\sum_{d \backslash n} \alpha_{m, d} f(d) g^{*}(n / d)=\sum_{d|n, d| m} f(d) g^{*}(n / d),
\end{aligned}
$$

which proves Theorem 3 .

ExAmples. For the special case $g=\mu$ we have $g^{*}=I$ so (14) becomes

$$
\sum_{d \backslash n} S_{f, \mu}(m, d)=\sum_{d \backslash(m, n)} f(d)[d / n]=\alpha_{m, n} f(n)=\left\{\begin{array}{lll}
f(n) & \text { if } & n \mid m \\
0 & \text { if } & n \nmid m .
\end{array}\right.
$$

When $f(n)=n$ this gives a formula of Von Sterneck ([7], p. 825),

$$
\sum_{d i n} \Phi(m, d)=\left\{\begin{array}{lll}
n & \text { if } & n \mid m \\
0 & \text { if } & n \nmid m
\end{array}\right.
$$

The type of argument used to prove Theorem 3 also gives the following more geneneral reseult.

THEOREM 4. For any arithmetical function $a$, let $B=g * a$. Then for $m \geqq 1, n \geqq 1$ we have

$$
\sum_{d \mid n} S_{f, g}(m, d) a(n / d)=\sum_{d \mid(m, n)} f(d) B(n / d) .
$$

If $g(1) \neq 0$ and $a=g^{-1}$, then $B=I$ and (16) reduces to

$$
\sum_{d \mid n} S_{f, g}(m, d) g^{-1}(n / d)=\alpha_{m, n} f(n)=\left\{\begin{array}{lll}
f(n) & \text { if } & n \mid m \\
0 & \text { if } & n \nmid m
\end{array} .\right.
$$

When $g=\mu$ this is the same as formula (15). 
ExAmPles. When $g=\mu$ and $a(n)=n$, then $B=\mu * a=\varphi$ and (16) implies

$$
\sum_{d \backslash n} d S_{f, \mu}\left(m, \frac{n}{d}\right)=\sum_{d \mid(m, n)} f(d) \varphi(n / d) .
$$

When $f(n)=n$ this gives the following identity for Von Sterneck's function,

$$
\sum_{d \backslash n} d \Phi(m, n / d)=\sum_{d\rfloor(m, n)} d \varphi(n / d)
$$

4. Partial sums involving the functions $S_{f, g}(m, k)$. The theorems of this section deal with sums whose terms include the numbers $S_{f, g}(m, k)$ where one of $m$ or $k$ is fixed and the other ranges over consecutive integers. First we introduce some notation.

As in the previous section we denote by $a^{*}$ the divisor sum

$$
a^{*}(n)=\sum_{d ! n} a(d),
$$

where $a$ is any arithmetical function. For real $x \geqq 1$ we also write

$$
a^{\wedge}(x)=\sum_{n \leqq x} a(n),
$$

and we put $a^{\wedge}(x)=0$ if $x<1$. We shall make use of the following lemma.

Lemma. For any two arithmetical functions $a$ and $b$ we have

$$
\sum_{n \leqq x}(a * b)(n)=\sum_{n \leqq x} a(n) b^{\wedge}(x / n) .
$$

Proof. We have

$$
\sum_{n \leqq x}(a * b)(n)=\sum_{n \leqq x} \sum_{d i n} a(d) b(n / d) .
$$

If $d \mid n$ we can write $n=q d$ and we obtain

$$
\sum_{n \leqq x} \sum_{d \uparrow n} a(d) b(n / d)=\sum_{d \leqq x} a(d) \sum_{q \leqq x / d} b(q)=\sum_{d \leqq x} a(d) b^{\wedge}(x / d) .
$$

THEOREM 5. For $k \geqq 1, n \geqq 1$, and any arithmetical function a we have

$$
\sum_{m=1}^{n} S_{f, g}(m, k) a^{\wedge}(n / m)=\sum_{d ! k, d \leqq n} f(d) g(k / d)\left(\alpha^{*}\right)^{\wedge}(n / m)
$$

Proof. We use the lemma, then Equation (8), and then the lemma once more to get 


$$
\begin{aligned}
\sum_{m=1}^{n} S_{f, g}(m, k) a^{\wedge}(n / m) & =\sum_{m=1}^{n}\left(h_{k} * \mu^{-1} * a\right)(m)=\sum_{m=1}^{n}\left(h_{k} * a^{*}\right)(m) \\
& =\sum_{m=1}^{n} h_{k}(m)\left(a^{*}\right)^{\wedge}(n / m)=\sum_{m=1}^{n} \alpha_{k, m} f(m) g(k / m)\left(a^{*}\right)^{\wedge}(n / m),
\end{aligned}
$$

which proves (17).

ExAmples. If we take $a=I$, then $a^{\wedge}(x)=1$ for all $x \geqq 1$ and $a^{*}(n)=1$ for all $n$, so $\left(a^{*}\right)^{\wedge}(x)=[x]$, and Theorem 5 becomes

$$
\sum_{m=1}^{n} S_{f, g}(m, k)=\sum_{d \mid k, d \leqq n} f(d) g(k / d)[n / d]
$$

If $k \mid n$, say $n=q k$, then every divisor $d$ of $k$ is $\leqq n$ and (18) takes the form

$$
\sum_{m=1}^{q k} S_{f, g}(m, k)=q k \sum_{d \backslash k} \frac{f(d)}{d} g\left(\frac{k}{d}\right) .
$$

For the special case $f(n)=n$ this gives us

$$
\sum_{m=1}^{q k} S_{f, g}(m, k)=q k g^{*}(k)
$$

In particular, when $g=\mu$ we obtain the following formula of Von Sterneck ([7], p. 825):

$$
\sum_{m=1}^{q k} \Phi(m, k)=q k \sum_{d \backslash k} \mu(d)= \begin{cases}q & \text { if } k=1 \\ 0 & \text { if } k>1\end{cases}
$$

THEOREM 6. For $m \geqq 1, n \geqq 1$ and any arithmetical function a we have

$$
\sum_{k=1}^{n} S_{f, g}(m, k) a^{\wedge}(n / k)=\sum_{d \mid m, d \leqq n} f(d)(g * a)^{\wedge}(n / d)
$$

Proof. This time we use the lemma in conjunction with (9) to obtain

$$
\begin{aligned}
\sum_{k=1}^{n} S_{f, g}(m, k) a^{\wedge}(n / k) & =\sum_{k=1}^{n}\left(w_{m} * g * a\right)(k)=\sum_{k=1}^{n} w_{m}(k)(g * a)^{\wedge}(n / k) \\
& =\sum_{k=1}^{n} \alpha_{m, k} f(k)(g * a)^{\wedge}(n / k)=\sum_{k \mid m, k \leqq n} f(k)(g * a)^{\wedge}(n / k) .
\end{aligned}
$$

This proves Theorem 6.

ExAmples. If $a=g^{-1}$ we have $g * a=I$ and $I^{\wedge}(x)=1$ for all $x \geqq$ 1, so Theorem 6 gives us the formula 


$$
\sum_{k=1}^{n} S_{f, g}(m, k)\left(g^{-1}\right)^{\wedge}(n / k)=\sum_{d \mid m, d \leqq n} f(d)
$$

In particular, if $g=\mu$ then $\left(g^{-1}\right)^{\wedge}(x)=[x]$ and (20) becomes

$$
\sum_{k=1}^{n}\left[\frac{n}{k}\right] S_{f, \mu}(m, k)=\sum_{d \mid m, d \leqq n} f(d) .
$$

When $f(n)=n$ this gives a theorem of Nicol ([6], p. 965),

$$
\sum_{k=1}^{n}\left[\frac{n}{k}\right] \Phi(m, k)=\sum_{d \mid m, d \leqq n} d=\sigma(m, n) .
$$

Here $\sigma(m, n)$ is the sum of the divisors of $m$ which are $\leqq n$.

If we take $a=I$, then $g * a=g$ and Theorem 6 becomes

$$
\sum_{k=1}^{n} S_{f, g}(m, k)=\sum_{d \mid m, d \leqq n} f(d) g^{\wedge}(n / d) .
$$

In particular, if $f(n)=n$ and $g=\mu$ we obtain the following formula for the sum of the $m$ th powers of all the primitive $k$ th roots of unity for $k=1,2, \cdots, n$ :

$$
\sum_{k=1}^{n} c_{k}(m)=\sum_{d \mid m, d \leqq n} d \mu^{\wedge}(n / d) .
$$

When $n=m$ this becomes

$$
\sum_{k=1}^{m} c_{k}(m)=\sum_{d \backslash m} d \mu^{\wedge}(m / d) .
$$

The right-hand member of (22) has the form $\left(f * \mu^{\wedge}\right)(m)$ where $f(n)=$ $n$. Since $f^{-1}(n)=n \mu(n)$, inversion of (22) gives us the following formula for the partial sums of the Möbius function:

$$
\sum_{n=1}^{m} \mu(n)=m \sum_{d \mid m} \frac{\mu(m / d)}{d} \sum_{k=1}^{d} c_{k}(d) .
$$

THEOREM 7. For $k \geqq 1, n \geqq 1$ and any completely multiplicative function a we have

$$
\sum_{m=1}^{n} a(m) S_{f, g}(m, k)=\sum_{d \mid k, d \leq n} a(d) f(d) g(k / d) a^{\wedge}(n / d) .
$$

Proof. We use formula (8) to obtain

$$
\begin{aligned}
\sum_{m=1}^{n} a(m) S_{f, g}(m, k) & =\sum_{m=1}^{n} a(m) \sum_{d \mid m} h_{k}(d)=\sum_{d=1}^{n} a(d) h_{k}(d) \sum_{q \leqq n / d} a(q) \\
& =\sum_{d=1}^{n} a(d) h_{k}(d) a^{\wedge}(n / d)=\sum_{d \mid k, d \leqq n} a(d) f(d) g(k / d) a^{\wedge}(n / d) .
\end{aligned}
$$


This proves Theorem 7.

ExAmples. If $a(n)=n$ then $a^{\wedge}(x)=[x]([x]+1) / 2$ and Theorem 7 gives us

$$
\sum_{m=1}^{n} m S_{f, g}(m, k)=\frac{1}{2} \sum_{d \mid k, d \leqq n} d f(d) g(k / d)[n / d]([n / d]+1) .
$$

If $k \mid n$, say $n=q k$, then $[n / d]=q k / d$ if $d \mid k$ and (24) becomes

$$
\sum_{m=1}^{q k} m S_{f, g}(m, k)=\frac{1}{2} q^{2} k^{2} \sum_{d \mid k} \frac{f(d)}{d} g(k / d)+\frac{1}{2} q k \sum_{d \nmid k} f(d) g(k / d) .
$$

When $g=\mu$ and $f(n)=n$ we obtain the formula

$$
\sum_{m=1}^{q k} m \Phi(m, k)=\frac{1}{2} q^{2} k^{2} I(k)+\frac{1}{2} q k \rho(k)= \begin{cases}\frac{1}{2} q(q+1) & \text { if } \quad k=1, \\ \frac{1}{2} q k \varphi(k) & \text { if } \quad k>1 .\end{cases}
$$

When $q=1$ this gives Theorem III of Nicol and Vandiner ([7], p. 830).

In Theorem 5 of [1] it was shown that for $R(s)>1$ we have

$$
\sum_{m=1}^{\infty} \frac{S_{f, g}(m, k)}{m^{s}}=\zeta \underset{d \mid k}{\zeta} \sum f(d) g(k / d) d^{-s}
$$

where $\zeta(s)=\sum_{n=1}^{\infty} n^{-s}$. Theorem 7 leads to another proof of this formula and also gives an estimate for the growth of the partial sums of the series on the left. Taking $a(m)=m^{-s}$, and putting $\sigma=R(s)$, we have (see [2], p. 618)

$$
a^{\wedge}(x)=\sum_{m \leqq x} \frac{1}{m^{s}}=\frac{x^{1-s}}{1-s}+\zeta(s)+O\left(x^{-\sigma}\right) .
$$

This formula is valid for all complex $s \neq 1$ with $\sigma>0$. Using this in Theorem 7 and taking $n=q k$, we obtain the formula

$$
\begin{aligned}
\sum_{m=1}^{g k} \frac{S_{f, g}(m, k)}{m^{s}}= & \frac{q^{1-s} k^{1-s}}{1-s} \sum_{d \backslash k} f(d) g(k / d) d^{-1} \\
& +\zeta(s) \sum_{d \backslash k} f(d) g(k / d) d^{-s}+O\left(q^{-\sigma} k^{-\sigma} \sum_{d \backslash k}|f(d) g(k / d)|\right) .
\end{aligned}
$$

If $\sigma>1$, the terms on the right which contain $q$ approach 0 as $q \rightarrow \infty$ and we obtain (25).

For the special case $f(d)=d, g=\mu$, we have

$$
\sum_{d i k} f(d) g(k / d) d^{-1}=\sum_{d \backslash k} \mu(k / d)=0 \quad \text { if } \quad k>1 .
$$


In this case the first term on the right of (27) vanishes if $k>1$ and the third term approaches 0 as $q \rightarrow \infty$ when $\sigma>0$. This gives a celebrated formula of Ramanujan ([9], p. 199)

$$
\sum_{m=1}^{\infty} \frac{c_{k}(m)}{m^{s}}=\zeta(s) \sum_{d \backslash k} d^{1-s} \mu(k / d)
$$

valid for $k>1$ and all complex $s \neq 1$ with $\sigma>0$.

For $s=1$ we have, instead of (26), the estimate

$$
a^{\wedge}(x)=\sum_{m \leqq x} \frac{1}{m}=\log x+C+O\left(\frac{1}{x}\right),
$$

where $C$ is Euler's constant. Using this in Theorem 7 with $n=q k$ we obtain the formula

$$
\begin{aligned}
\sum_{m=1}^{q k} \frac{S_{f, g}(m, k)}{m}= & (C+\log k+\log q) \sum_{d \backslash k} f(d) d^{-1} g(k / d) \\
& -\sum_{d \backslash k} f(d) d^{-1} g(k / d) \log d+O\left(q^{-1} k^{-1} \sum_{d \backslash k}|f(d) g(k / d)|\right) .
\end{aligned}
$$

This shows that the series $\sum_{m=1}^{\infty} S_{f, g}(m, k) / m$ converges if and only if the coefficient of $\log q$ vanishes, that is, if and only if

$$
\sum_{d \mid k} f(d) d^{-1} g(k / d)=0
$$

in which case we obtain

$$
\sum_{m=1}^{\infty} \frac{S_{f, g}(m, k)}{m}=-\sum_{d \backslash k} f(d) d^{-1} g(k / d) \log d .
$$

When $f(d)=d$ and $g=\mu$, Equation (28) is satisfied for $k>1$ and we obtain another formula of Ramanujan ([9], p. 199),

$$
\sum_{m=1}^{\infty} \frac{c_{k}(m)}{m}=-\sum_{d \mid k} \mu(k / d) \log d=-\Lambda(k),
$$

where $k>1$ and $\Lambda(k)$ is Mangoldt's function.

THEOREM 8. For $m \geqq 1, n \geqq 1$ and any completely multiplicative function a we have

$$
\sum_{k=1}^{n} a(k) S_{f, g}(m, k)=\sum_{d \mid m, d \leq n} a(d) f(d)(a g)^{\wedge}(n / d),
$$

where $(a g)^{\wedge}(r)=\sum_{d \leqq r} a(d) g(d)$.

Proof. We use formula (9) to obtain 


$$
\begin{aligned}
\sum_{k=1}^{n} a(k) S_{f, g}(m, k) & =\sum_{k=1}^{n} a(k) \sum_{d \nmid k} w_{m}(d) g(k / d)=\sum_{d=1}^{n} a(d) w_{m}(d) \sum_{q \leq n / d} a(q) g(q) \\
& =\sum_{d=1}^{n} a(d) \alpha_{m, d} f(d)(a g)^{\wedge}(n / d)=\sum_{d \mid m, d \leqq n} a(d) f(d)(a g)^{\wedge}(n / d) .
\end{aligned}
$$

EXAMPLES. In Theorem 5 of [1] it was shown that

$$
\sum_{k=1}^{\infty} \frac{S_{f, g}(m, k)}{k^{s}}=G(s) \sum_{d \mid m} f(d) d^{-s}
$$

for each $s$ for which the Dirichlet series $G(s)=\sum_{r=1}^{\infty} g(r) r^{-s}$ is convergent. We ban derive this also from Theorem 8 and obtain a formula for the partial sums of the series on the left.

Taking $a(n)=n^{-s}$ we have

$$
(a g)^{\wedge}(x)=\sum_{r \leqq x} g(r) r^{-s}=G(s)-\sum_{n>x} g(r) r^{-s}
$$

if the series for $G(s)$ converges. Using this in Theorem 8 and taking $n=q m$ we find

$$
\sum_{k=1}^{q m} \frac{S_{f, g}(m, k)}{k^{s}}=G(s) \sum_{d \backslash m} f(d) d^{-s}-\sum_{d \backslash m} f(d) d^{-s} \sum_{r>q m / d} g(r) r^{-s} .
$$

Letting $q \rightarrow \infty$ we obtain (29).

In the special case with $f(d)=d, g=\mu$, we have

$$
G(s)=\sum_{r=1}^{\infty} \frac{\mu(r)}{r^{s}}=\frac{1}{\zeta(s)} \text { for } R(s)>1 .
$$

This series also converges for $s=1$ and $G(1)=0$. Also,

$$
\sum_{d \backslash m} f(d) d^{-s}=\sum_{d \backslash m} d^{1-s}=\sigma_{1-s}(m) .
$$

In this case (29) gives another formula of Ramanujan ([9], p. 185)

$$
\sum_{k=1}^{\infty} \frac{c_{k}(m)}{k^{s}}=\frac{\sigma_{1-s}(m)}{\zeta(s)}
$$

valid for $R(s)>1$ and also for $s=1$.

5. An extension of Smith's determinant. For any arithmetical function $f$, let

$$
f^{*}(r)=\sum_{d \mid r} f(d)
$$

and let $A=\left[f^{*}(m, k)\right]$ be the $n \times n$ matrix whose $m, k$ entry is the value of $f^{*}$ at the greatest common divisor $(m, k)$ of $m$ and $k$. H. J. S. Smith [10] proved the determinant formula 


$$
\operatorname{det} A=f(1) f(2) \cdots f(n) \text {. }
$$

Dickson ([3], pp. 122-129) reports on several papers by Catalan, Cesaro, Gegenbauer, Mansion, and others, devoted to proofs and extensions of (30). D. H. Lehmer [5] also generalized (30) to higher-dimensional determinants.

A simple proof of (30), suggested by Pólya and Szegö (see [8], p. 330), is based on the observation that $A=B C^{t}$, where $B$ and $C$ are lower triangular $n \times n$ matrices with $\operatorname{det} B=f(1) f(2) \cdots f(n)$ and $\operatorname{det} C=1$. This section extends this proof to provide the following new generalization of $(30)$.

TheOREM 9. Let $A$ be the $n \times n$ matrix whose $m, k$ entry is $S_{f, g}(m, k)$. Then we have

$$
\operatorname{det} A=f(1) f(2) \cdots f(n) g(1)^{n} .
$$

Proof. We express the $n \times n$ matrix $A$ as a product, $A=$ $B(f) C(g)^{t}$, where $B(f)$ and $C(g)$ are lower triangular $n \times n$ matrices given by

$$
B(f)=\left[\alpha_{m, k} f(m)\right], \quad C(g)=\left[\alpha_{m, k} g(m / k)\right] .
$$

Then $m, k$ entry of $B(f) C(g)^{t}$ is equal to

$$
\sum_{r=1}^{n} \alpha_{m, r} f(r) \alpha_{k, r} g(k / r)=\sum_{r|m, r| k} f(r) g(k / r)=S_{f, g}(m, k),
$$

so $A=B(f) C(g)^{t}$, as asserted. Since $\operatorname{det} B(f)=f(1) \cdots f(n)$ and $\operatorname{det} C(g)=g(1)^{n}$, we obtain (31).

EXAMPLE. When $f(n)=n$ and $g=\mu$, we obtain the following formula for the determinant of the $n \times n$ matrix whose $m, k$ entry is the Ramanujan sum $c_{k}(m)$ :

$$
\operatorname{det}\left[c_{k}(m)\right]=n ! .
$$

\section{REFERENCES}

1. Douglas R. Anderson and T. M. Apostol, The evaluation of Ramanujan's sum and generalizations, Duke Math. J., 20 (1953), 211-216.

2. Tom M. Apostol, Calculus, Vol. II, Second Edition, Blaisdell, Waltham, Mass., 1969.

3. L. E. Dickson, History of the Theory of Numbers, Vol. 2, Chelsea, New York, 1952.

4. O. Hölder, Zur Theorie der Kreisteilungsgleichung $K_{m}(x)=0$, Prace Matematyczno Fizyczne, 43 (1936), 13-23.

5. D. H. Lehmer, The $p$ dimensional analogue of Smith's determinant, American Math. Monthly, 37 (1930), 294-296.

6. Charles A. Nicol, On restricted partitions and a generalization of the Euler $\varphi$ number and the Moebius function, Proc. Nat. Acad. Sci., 39 (1953), 963-968. 
7. C. A. Nicol and H. S. Vandiver, A Von Sterneck arithmetical function and restricted partitions with respect to a modulus, Proc. Nat. Acad. Sci., 40 (1954), 825-835.

8. G. Pólya and G. Szegö, Aufgaben und Lehrsätze aus der Analysis, vol. II, Springer, Berlin, 1925, VIII Abschn., Kap. 1, Nr. 33, p. 330.

9. Srinivasa Ramanujan, On certain trigonometrical sums and their applications in the theory of numbers, Trans. Cambridge Phil. Soc., 22 (1918), 259-276.

10. H. J. S. Smith, On the value of a certain arithmetical determinant, Proc. London Math. Soc., 7 (1875-6), 208-212.

11. R. Daublebsky Von Sterneck, Ein Analogon zur additiven Zahlentheorie, Sitzber, Akad. Wiss. Wien, Math.-Naturw. Klasse, vol. 111 (Abt. Ila) (1902), 1567-1601.

Received February 4, 1971.

California Institute of Technology 



\section{PACIFIC JOURNAL OF MATHEMATICS}

\section{EDITORS}

\author{
H. SAMELSON \\ Stanford University \\ Stanford, California 94305

\section{R. HoвBY} \\ University of Washington \\ Seattle, Washington 98105
}

\section{J. DugundJI}

Department of Mathematics University of Southern California Los Angeles, California 90007

\author{
RichaRd ARENS \\ University of California \\ Los Angeles, California 90024
}

\section{ASSOCIATE EDITORS}
E. F. BECKENBACH
B. H. NEUMANN
F. WOLF
K. YoshidA

\section{SUPPORTING INSTITUTIONS}

\author{
UNIVERSITY OF BRITISH COLUMBIA \\ CALIFORNIA INSTITUTE OF TECHNOLOGY \\ UNIVERSITY OF CALIFORNIA \\ MONTANA STATE UNIVERSITY \\ UNIVERSITY OF NEVADA \\ NEW MEXICO STATE UNIVERSITY \\ OREGON STATE UNIVERSITY \\ UNIVERSITY OF OREGON \\ OSAKA UNIVERSITY
}

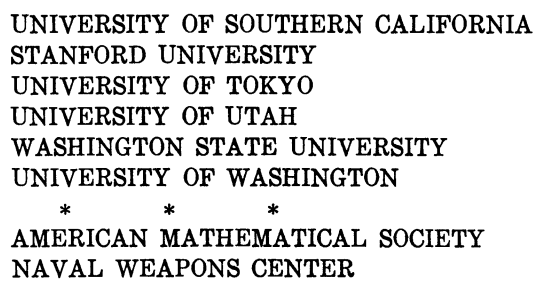

The Supporting Institutions listed above contribute to the cost of publication of this Journal, but they are not owners or publishers and have no responsibility for its content or policies.

Mathematical papers intended for publication in the Pacific Journal of Mathematics should be in typed form or offset-reproduced, (not dittoed), double spaced with large margins. Underline Greek letters in red, German in green, and script in blue. The first paragraph or two must be capable of being used separately as a synopsis of the entire paper. The editorial "we" must not be used in the synopsis, and items of the bibliography should not be cited there unless absolutely necessary, in which case they must be identified by author and Journal, rather than by item number. Manuscripts, in dup icate if possible, may be sent to any one of the four editors. Please classify according to the scheme of Math. Rev. Index to Vol. 39. All other communications to the editors should be addressed to the managing editor, Richard Arens, University of California, Los Angeles, California, 90024.

50 reprints are provided free for each article; additional copies may be obtained at cost in multiples of 50 .

The Pacific Journal of Mathematics is published monthly. Effective with Volume 16 the price per volume (3 numbers) is $\$ 8.00$; single issues, $\$ 3.00$. Special price for current issues to individual faculty members of supporting institutions and to individual members of the American Mathematical Society: $\$ 4.00$ per volume; single issues $\$ 1.50$. Back numbers are available.

Subscriptions, orders for back numbers, and changes of address should be sent to Pacific Journal of Mathematics, 103 Highland Boulevard, Berkeley, California, 94708.

PUBLISHED BY PACIFIC JOURNAL OF MATHEMATICS, A NON-PROFIT CORPORATION

Printed at Kokusai Bunken Insatsusha (International Academic Printing Co., Ltd.), 270, 3-chome Totsuka-cho, Shinjuku-ku, Tokyo 160, Japan. 


\section{Pacific Journal of Mathematics}

\section{Vol. 41, No. 2 December, 1972}

Tom M. (Mike) Apostol, Arithmetical properties of generalized Ramanujan sums .......................................... 281

David Lee Armacost and William Louis Armacost, On p-thetic groups ........ 295

Janet E. Mills, Regular semigroups which are extensions of groups .......... 303

Gregory Frank Bachelis, Homomorphisms of Banach algebras with minimal ideals ................................................ 307

John Allen Beachy, A generalization of injectivity .................. 313

David Geoffrey Cantor, On arithmetic properties of the Taylor series of rational functions. II.........................................

Václáv Chvátal and Frank Harary, Generalized Ramsey theory for graphs. III.

Small off-diagonal numbers .................................. 335

Frank Rimi DeMeyer, Irreducible characters and solvability of finite groups . . . . 347

Robert P. Dickinson, On right zero unions of commutative semigroups........ 355

John Dustin Donald, Non-openness and non-equidimensionality in algebraic

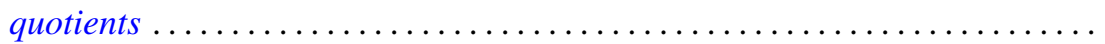

John D. Donaldson and Qazi Ibadur Rahman, Inequalities for polynomials with a prescribed zero ........................................ 375

Robert E. Hall, The translational hull of an $N$-semigroup ................ 379

John P. Holmes, Differentiable power-associative groupoids.............. 391

Steven Kenyon Ingram, Continuous dependence on parameters and boundary data for nonlinear two-point boundary value problems .

Robert Clarke James, Super-reflexive spaces with bases ..........

Gary Douglas Jones, The embedding of homeomorphisms of the plane in

continuous flows...............................

Mary Joel Jordan, Period $H$-semigroups and $t$-semisimple periodic

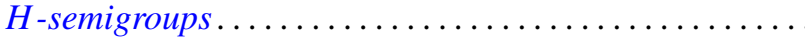

Ronald Allen Knight, Dynamical systems of characteristic 0

Kwangil Koh, On a representation of a strongly harmonic ring by sheaves...

Hui-Hsiung Kuo, Stochastic integrals in abstract Wiener space. ..

Thomas Graham McLaughlin, Supersimple sets and the problem of extending a

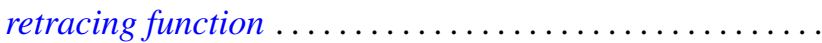

William Nathan, Open mappings on 2-manifolds .

M. J. O'Malley, Isomorphic power series rings

Sean B. O'Reilly, Completely adequate neighborhood systems and metrization

Qazi Ibadur Rahman, On the zeros of a polynomial and its derivative...

Russell Daniel Rupp, Jr., The Weierstrass excess function ..

Hugo Teufel, A note on second order differential inequalities and functional

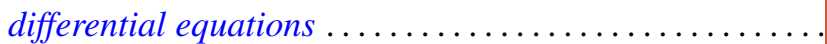

M. J. Wicks, A general solution of binary homogeneous equations over free 